\title{
REPRODUCTIVE BIOLOGY OF A MEDICINAL ORCHID SATYRIUM NEPALENSE (ORCHIDACEAE) IN THE NEPALESE HIMALAYAS
}

\author{
Babu Ram Paudel ${ }^{1 *}$ and Brihaspati Poudel ${ }^{2}$ \\ ${ }^{1}$ Department of Botany, Prithvi Narayan Campus, Tribhuvan University, Pokhara, Nepal \\ ${ }^{2}$ College of Ecology and Environmental Sciences, Yunnan University, Kunming, China \\ *For correspondence: brp2033@gmail.com
}

\begin{abstract}
Satyrium nepalense is a terrestrial medicinal orchid of the Himalayas. Although, the ecology and medicinal uses of this orchid is well known, little is known about its reproductive mechanism. In this study, we explored the natural history and reproductive biology of $S$. nepalense and tested their potential spatial variation. We observed the floral visitors and natural fruit set of $S$. nepalense at its two populations. We found that, despite the presence of outcrossing features, this orchid did not receive any diurnal floral visitors. The natural fruit set was very high $(>90.0 \%)$ at both the sites, suggesting the possibility of autonomous selfing. This finding may have important implication for the further study to explore how autonomous selfing evolves in a member of the predominately outcrossing genus. Moreover, the finding of this study provides important insight to develop efficient protocol for its conservation.
\end{abstract}

Key words: Autonomous selfing, conservation, floral traits, orchids, pollination

\section{INTRODUCTION}

Orchids, the members of the family Orchidaceae, are well known for possessing exquisite flowers exhibiting great diversity in shape, size, color, fragrance, and texture (Dressler, 1981). This diversity in floral architecture is often considered the result of adaptation towards different pollination mechanisms (Darwin, 1862; Van der Cingel, 2001). Accordingly, they exhibit complex pollination strategies including various curious mechanisms of autonomous selfing, specialized biotic pollination assisted either by reward or mimicry leading to deceptive pollination, and/or various forms of abiotic pollination (Johnson, 1996; Johnson et al., 2011). However, most orchids evolve mechanism to prevent self-pollination and thus reproduce through a highly specialized pollinator mediated outcrossing. Thus, the natural reproduction in orchids often relies upon the availability of pollinators (Catling 1990; Peter 2009). This high level of pollinator specialization may often lead to reproductive failure if the phenology/ distribution/ habitat of pollinators are disturbed by multifarious factors including anthropogenic disturbances. In alpine habitat, the availability of pollinator is limited by several factors and thus the alpine orchids may suffer from high level of pollen limitation (Catling, 1990, Johnson and Steiner, 2003; Tremblay et al., 2005). As a result, like other alpine plants, orchids in the high Himalayas may evolve either abiotic pollination or autonomous selfing to assure their reproductive success. Studies have shown that $c a 400$ orchid (comprising ca 5\% orchid) species are known to reproduce via autonomous selfing while the cases of abiotic pollination are rare (Catling, 1990; Peter, 2009). Due to these 
specific pollination system of orchids, exploring the reproductive mechanism is an essential step in developing efficient protocol for their conservation.

The genus Satyrium, a twin-spurred orchid, is well known for possessing unsurpassed floral diversity particularly associated with pollinator mediated pollination systems (Van der Niet $e t$ al., 2005; Liltved and Johnson, 2012). Consistent with the floral traits, most members of the genus exhibit specialized biotic pollination system including bee, beetle, bird, butterfly, fly, and moth as the specialized pollinators (Johnson, 1996, 1997; Johnson et al., 2011). Satyrium nepalense D. Don like other members of the genus possesses the floral traits that are consistent with pollinator mediated pollination system. Thus, we anticipate pollinator mediated breeding system in this orchid. Although, the ecology and medicinal uses of this orchid have been studied by several authors, its reproductive biology is yet unexplored (Mishra and Saklani, 2012; Paudel and Poudel, 2019). In this study, we explored the natural history and reproductive biology of S. nepalense and tested their potential spatial variation.

\section{MATERIALS AND METHODS}

\section{Study species and study sites}

Satyrium nepalense is a terrestrial orchid distributed from the Indian subcontinent to South central China. In Nepal, it is distributed across the central, western and eastern parts of Nepal between the elevation of 1000-3200 m (Raskoti, 2009). It preferably grows in south/ east facing open meadows (Paudel and Poudel, 2019). It is a medium sized herb with 14-32 $\mathrm{cm}$ height, cylindrical stem and possesses 2-3 sheathing with sheath leaves. Flowering occurs from August to October with the peak blooming in early September. An individual plant produces a single erect spike (inflorescence) that usually bears 10-50 compactly arranged rose pink flowers (Fig.1) (Raskoti, 2009).

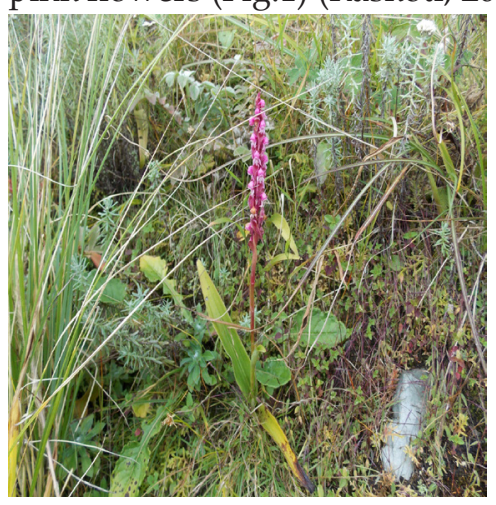

Figure 1: A flowering individual of S. nepalense at its natural habitat (Deorali Pass)

The study was conducted at two sites within the Annapurna conservation area. The first site (Deorali Pass) is a south facing meadow located just above Nangethati, Kaski while the second site is an east facing meadow close to the famous tourist destination Poonhill, Myagdi (Fig. 1). At both the sites, the plant grows mixed with another terrestrial orchid Herminium sp., several grasses, ferns, Pedicularis, Potentilla etc.

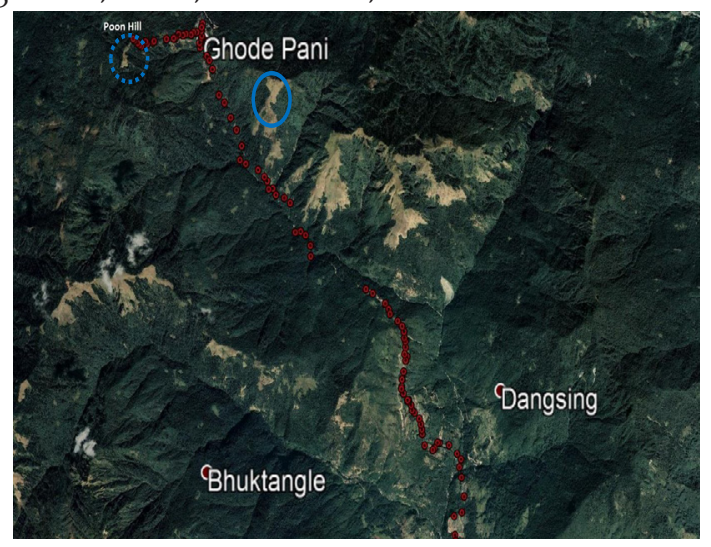

Figure 2: Study sites. Site included in solid circle indicates Deorali Pass while the site included within dotted circle indicates Poonhill area. Dotted red line indicates the trekking route to the study sites.

\section{Breeding system experiments}

At each sites, we observed the potential visitors to $S$. nepalense flowers for three consecutive days 
from 7:00 to $17: 00 \mathrm{hr}$. As this time interval is the peak foraging time of diurnal visitors, this observation includes all the potential diurnal floral visitors. Due to logistic constrain, however, wewere unable to observe the potential nocturnal visitors. To determine the potential mechanism of natural breeding system, at each site we randomly selected 25 flowering individuals. All the flowers of the experimental individuals were then counted, tagged, and left intact to allow fruit set. After about a month, fruits formed in each experimental individual were separately counted, and the percentage of fruit formed in each individual plant was assessed. We then tested the differences in fruit set percentage among the different plants at a site. Finally, the differences in fruit set percentage between the two sites were assessed. We used generalized linear models with binary distribution of errors to differentiate the fruit set percentage among the individual plants/between the sites. All the analyses were performed in $\mathrm{R}$ package (version 3.3.1) (R Core Team, 2015).

\section{RESULTS}

Our result revealed the absence of diurnal visitors to the flowers of $S$. nepalense, i.e., during our three days' survey at each site, we did not observe any visitors to the flowers of S. nepalense. We found that at both the sites more than $90 \%$ flowers at each inflorescence (individual plant) formed fruits. Our analysis revealed that the fruit set percentage among the different plants (inflorescences) within a site did not differ significantly $(\mathrm{P}>0.05)$. Similarly, the fruit set percentage did not differ significantly between the two sites $(\mathrm{P}>0.05)$. The average fruit set percentages at Deorali pass and Poonhill area were $93.45 \%$ and $90.43 \%$ respectively (Fig. 3).

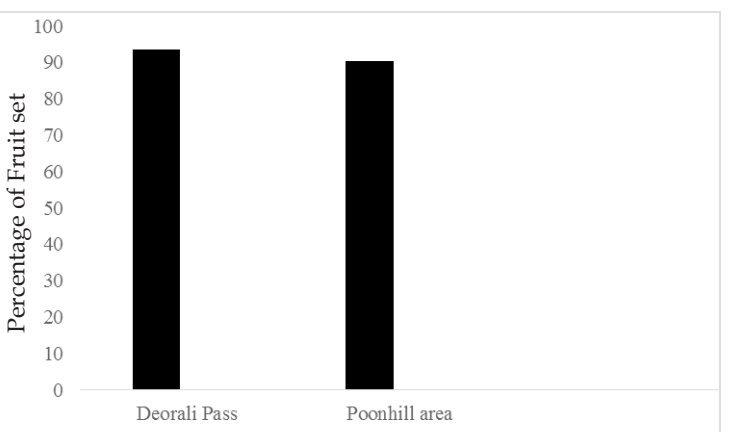

Figure 3: Natural fruit set percentage of $S$. nepalense at the two experimental sites.

\section{DISCUSSION}

Our result indicates that despite the presence of several outcrossing floral features, $S$. nepalense flowers lack diurnal floral visitors, yet the natural fruit set is very high. The high proportion of fruit set data suggest the possibility of autonomous selfing in this orchid. Although the fruit set success in this orchid could have been either via the contribution of nocturnal pollinator or via abiotic pollination mediated by air and water. However, our data does not support for either of the possibility. Under the condition of nocturnal pollination, usually the fruit set proportion could not be as high as the current data. Similarly, abiotic pollination assisted by wind or water also seems unlikely to occur because of the aggregation of pollen grains into pollinia that are not easily dislodged by rain and/or water (Suetsugu, 2019). Therefore, the reproduction in S. nepalense seems to occur through autonomous selfing. Our finding is consistent with the finding of Niet (2018) in S. rupestre. Given the absence of absence of autonomous selfing in most members of the genus, and the presence of outcrossing floral feature, why and how $S$. nepalense evolve autonomous selfing is an intriguing question for further research. Niet (2018) suggests that the presence of unusual column in the flowers facilitates autonomous selfing in S. rupestre. So, 
it suggests that S. nepalense may possess such a unique floral feature that facilitates autonomous slefing. However, our current limited data cannot provide strong evidence in favour of autonomous slefing in S. nepalense. Therefore, further study including the close observation of floral traits and manipulated pollination experiment would be required to shed light in the actual pollination mechanism of this highly valued medicinal orchid of the Himalayas.

\section{REFERENCES}

Catling, P. M. (1990). Auto-pollination in the Orchidaceae. Orchid Biology: Reviews and Perspectives. 5:121-158.

Darwin, C.R. (1862). On the various contrivances by which British and foreign orchids are fertilized by insects. John Murray, London.

Dressler, R.L. (1981). The orchids: natural history and classification. Harvard University, Cambridge, USA.

Johnson, S.D. (1996). Bird pollination in South African species of Satyrium (Orchidaceae). Plant Systematics and Evolution. 203: 91-98.

Johnson, S.D. (1997). Insect pollination and floral mechanisms in South African species of Satyrium (Orchidaceae). Plant Systematics and Evolution. 204: 195-206.

Johnson, S.D., C.I. Peter, A.G. Ellis, E. Boberg, C. Botes, T. Van der Niet (2011). Diverse pollination systems of the twin-spurred orchid genus Satyrium in African grasslands. Plant Systematics and Evolution. 292: 95103.

Johnson, S.D. and K.E. Steiner (2003). Specialized pollination systems in southern Africa. South African Journal of Science 99: 345-348.

Liltved, W. and S. Johnson (2012). The Cape Orchids; A Regional Monograph of the Orchids of the Cape Floristic Region. Sandstone Editions, Noordhoek.
Mishra A.P. and S. Saklani (2012). Satyrium nepalense: a rare medicinal orchid of western himalaya (india); phytochemical screening, antimicrobial evaluation and conservation studies. Indonesian J. Pharm. 23 (3): 162 - 170

Paudel B.R. and B. Poudel (2019). Ecological study of a medicinal orchid (Satyrium nepalense) in an alpine meadow. Himalayan Biodiversity.7:1-5

Peter, C. I. (2009). Pollinators, floral deception and evolutionary processes in Eulophia (Orchidaceae) and its allies. Dissertation, University of KwaZulu-Natal, KwaZuluNatal, South Africa.

R Core Team (2015). R: a language and environment for statistical computing. $\mathrm{R}$ Foundation for Statistical Computing, Vienna, Austria.

Raskoti, B.B (2009). The orchids of Nepal. Quality Printers, Nepal.

Suetsugu, K. (2019). Rain-triggered selfpollination in Liparis kumokiri, an orchid that blooms during the rainy season. Ecology. 100(7): e02683. 10.1002/ ecy.2683

Tremblay, R.L., J.D. Ackerman, J.K. Zimmerman, R.N. Calvo (2005). Variation in sexual reproduction in orchids and its evolutionary consequences: a spasmodic journey to diversification. Biological Journal of the Linnean Society. 84: 1-54.

Van der Cingel, N.A. (2001). An Atlas of Orchid Pollination: America, Africa, Asia and Australia. A. A. Balkema, Rotterdam.

Van der Niet, T., H.P. Linder, B. Bytebier, D.U. Bellstedt, (2005). Molecular markers reject monophyly of the subgenera of Satyrium (Orchidaceae). Systematic Botany.30: 263274. 